\title{
Diagnosing Machine Learning Pipelines with Fine-grained Lineage
}

\author{
Zhao Zhang \\ Texas Advanced Computing Center, \\ The University of Texas at Austin \\ zzhang@tacc.utexas.edu
}

\author{
Evan R. Sparks \\ Computer Science Division, \\ University of California, Berkeley \\ sparks@cs.berkeley.edu
}

\author{
Michael J. Franklin \\ Department of Computer Science, \\ University of Chicago \\ mjfranklin@uchicago.edu
}

\begin{abstract}
We present the Hippo system to enable the diagnosis of distributed machine learning (ML) pipelines by leveraging fine-grained data lineage. Hippo exposes a concise yet powerful API, derived from primitive lineage types, to capture fine-grained data lineage for each data transformation. It records the input datasets, the output datasets and the cell-level mapping between them. It also collects sufficient information that is needed to reproduce the computation. Hippo efficiently enables common ML diagnosis operations such as code debugging, result analysis, data anomaly removal, and computation replay. By exploiting the metadata separation and high-order function encoding strategies, we observe an $O\left(10^{3}\right)$ x total improvement in lineage storage efficiency vs. the baseline of cell-wise mapping recording while maintaining the lineage integrity. Hippo can answer the real use case lineage queries within a few seconds, which is low enough to enable interactive diagnosis of ML pipelines.
\end{abstract}

\section{KEYWORDS}

machine learning; diagnostics; fine-grained lineage

\section{ACM Reference format:}

Zhao Zhang, Evan R. Sparks, and Michael J. Franklin. 2017. Diagnosing Machine Learning Pipelines with Fine-grained Lineage. In Proceedings of HPDC '17, Washington, DC, USA, fune 26-30, 2017, 11 pages.

DOI: http://dx.doi.org/10.1145/3078597.3078603

\section{INTRODUCTION}

Machine learning frameworks are increasingly popular as practitioners and researchers can quickly build applications (referred to as ML pipelines or pipelines in the rest of this paper) with a highlevel programming language to pipeline data preparation, feature extraction, model training, and prediction. Among these systems, Scikit-learn [32] is a single-computer-based framework while TensorFlow [17], MLlib [25], SystemML [16], and KeystoneML [37] focus on a distributed environment.

In practice, to obtain and deploy a working model, users often need to try a number of different datasets, featurization techniques, training parameters, and model families. When experimenting with

Permission to make digital or hard copies of all or part of this work for personal or classroom use is granted without fee provided that copies are not made or distributed for profit or commercial advantage and that copies bear this notice and the full citation on the first page. Copyrights for components of this work owned by others than ACM must be honored. Abstracting with credit is permitted. To copy otherwise, or republish, to post on servers or to redistribute to lists, requires prior specific permission and/or a fee. Request permissions from permissions@acm.org.

HPDC '17, Washington, DC, USA

(C) 2017 ACM. 978-1-4503-4699-3/17/06 ..\$15.00

DOI: http://dx.doi.org/10.1145/3078597.3078603 these options, users frequently analyze results together with the corresponding input data, locate code segments responsible for unexpected outcomes, or rerun the computation with the removal of suspicious data anomalies.

As a concrete example, consider the task of light source extraction in astronomy: this pipeline reads telescope images and produces a list of potential celestial objects with their positions, luminous flux, estimated error, and other information. One routine quality check involves tracing those abnormally bright objects in the result to the contributing pixels in the original input images. Then with further mathematical analysis or human intervention, this user must be able to validate if these abnormally bright objects are caused by a bad charge-coupled device (CCD) pixel. Once an erroneous pixel is confirmed, the user may then want to find out how this erroneous data item propagates to the rest of the results. Such an inspection involves two types of operations: forward queries that trace the resulting output cells given an input cell and backward queries that trace the contributing input cells given an output cell. These two query types are made possible by the celllevel consumption-production dependency mapping. This cell-wise dependency is also referred to as fine-grained data lineage.

Given that such diagnosis is a commonly repeated work pattern, it is natural to add system support to capture and work with fine-grained data lineage directly in an ML framework. In this paper, we describe the design of a distributed ML pipeline diagnostic system that leverages fine-grained data lineage. Compared to coarse-grained lineage at file or relational table metadata level, finegrained lineage is at the data structure cell level, e.g., elements in a matrix or attributes in a record. We assume an ML framework with a programming model that abstracts a pipeline as a chain of data transformations, such as KeystoneML, TensorFlow, and SystemML. In particular, KeystoneML builds on top of Apache Spark [44] and uses two building blocks: transformers and estimators. A transformer applies a unary function to data items and produce new ones. An estimator takes a collection of data items, feeds them to a training procedure, and produces a transformer. We choose this model because it is widely adopted and allows users to declare and instrument lineage capturing directly in the transformer. Our diagnostic system provides:

- A concise and flexible interface for users to declare and instrument lineage capturing

- Low overhead for capturing and storing lineage

- Interactive lineage query for pipeline diagnosis

Lineage tracking has been investigated in many contexts. Scientific workflow systems such as Chimera [13], Taverna [28], ESSW [15], Kepler [2], and Karma2 [36] can collect coarse-grained lineage at the level of files and computations. The Taverna 2 workflow system [38] proposes a fine-grained lineage model on the collection 
Table 1: Performance Penalty Reported by Existing Lineage Systems

\begin{tabular}{|c|c|c|}
\hline Project & Underlying System & Perf Penalty \\
\hline \hline RAMP [19] & Hadoop [4] & $16-76 \%$ \\
\hline Newt [22] & Hadoop [4] & $20-50 \%$ \\
\hline SubZero [42] & SciDB [7] & $50 \%-150 \%$ \\
\hline Titian [20] & Spark [44] & $10 \%-29 \%$ \\
\hline
\end{tabular}

element level. And the later work by Missier et al. [26] presents two mapping types between input and output as data collection cross-product and index-projection. In the context of distributed processing, RAMP [19, 31] and Newt [22] present solutions for MapReduce systems. Titian [20] captures Apache Spark's lineage at collection element level. Fine-grained lineage is also investigated in the filed of database and data warehouse by works [9, 10, 40, 42].

Unfortunately, these existing lineage tracking systems are not suitable for distributed ML frameworks for three reasons. First, coarse-grained lineage information collected by scientific workflow systems (e.g., Chimera) lacks the necessary detail of cell-level mapping. Even Titian only captures Spark lineage at collection element level, e.g., a matrix in a list, whereas ML pipeline diagnosis requires mapping at one level deeper on the matrix cells. Second, the two mapping types presented in the work of Missier et al. [26] partially address the input and output mapping in workflow computations. But they are not sufficient to cover the data transformation in ML pipelines. Third, the lineage capturing interface in other related systems is often designed with the underlying system operators (e.g., SQL operators for RDBMS, mappers and reducers in MapReduce systems, and input-stage-aggregation operators for Apache Spark.). In contrast, ML frameworks do not have such a set of well-defined low level operators, which makes it difficult to design a general lineage capturing interface. Other challenges of building an ML framework diagnostic system include the cost introduced by lineage capturing and the low latency requirement for lineage queries to enable interactive diagnosis. Table 1 shows the performance penalty incurred by lineage capturing in recent work.

Because ML pipeline builders have deep understanding of data transformations, we present a lineage capturing interface that lets users decide what data to capture and how to capture it. The lineage capturing interface is designed by formulating the cell level mapping of an ML pipeline as a sequence of multi-dimensional space transformations. This interface contains seven primitive mapping types: namely, collapse, flatten, identity, all, linear combination, geometry, and join. These seven primitive mappings cover $87 \%$ (39 of 45), 89\% (130 of 146), and 89\% (72 of 81) of the pre-defined data transformations in KeystoneML, TensorFlow, and SystemML, respectively. For transformations that are not covered, the lineage capturing interface allows users to specify customized mapping functions. A formal definition of the mapping types is in Section 4.

In this paper, we present Hippo $^{1}$, a fine-grained lineage capturing and serving system for distributed ML pipelines. Hippo exposes the lineage capturing interface by exploiting the seven primitive mapping types. It also provides the cell-level tracing functionality with a query interface so that users are able to find the contributing and resulting cells given a cell of interest. Hippo is also flexible so

\footnotetext{
${ }^{1}$ https://github.com/zhaozhang/keystone/tree/lineage-v4
}

that users can collect optional data inside a data transformation. These optional data include the input/output dataset, the transformation itself, the model, and the state parameter (e.g., a random seed). Hippo optimizes lineage data storage efficiency with techniques of data structure metadata(e.g., the size of each dimension of a space) separation and high-level function encoding. To speedup the query, Hippo employs the techniques of spatial index and a rule-based mapping and query reduction strategy.

To showcase the effectiveness of Hippo's design and optimization, we integrated Hippo with the open source KeystoneML distributed machine learning framework. We demonstrate the utility of Hippo by studying three distinct use cases enabled by fine-grained lineage. These use cases are: code validation, results inspection, and data cleaning. A detailed discussion of these three use cases is presented in Section 2.3. All these use cases are enabled by either fine-grained lineage query or by intermediate data reuse. Hippo's lineage capturing and serving functionalities alleviate users from repeatedly modifying the source code and executing the pipeline to obtain the necessary diagnosis information. Compared to the naive solution of materializing cell-wise mapping, Hippo's techniques (spatial index and high-order function) enable $2 \mathrm{x}-31 \mathrm{x}$ speedups at lineage query time. This new capability comes at the cost of modest performance overheads during pipeline training/execution. The overhead is measured in wall clock time, memory consumption, and storage space. For the three use cases, the wall clock time overhead for lineage capturing is $41 \%-61 \%$, which is comparable to other distributed lineage systems summarized in Table 1. Hippo increases memory usage by $31 \%-79 \%$, and the storage space for lineage information varies case by case from $\sim 100 \mathrm{MB}$ to $\sim 1 \mathrm{~TB}$.

In summary, we make the following contributions:

- We design a concise and powerful lineage capturing interface by formulating the cell-wise mapping in ML pipelines as multi-dimensional space transformations.

- We map many common ML operations such as featurization and labeling to the lineage capturing interface.

- We introduce a high-order function approach for describing geometry to reduce storage overhead. Meanwhile, we use spatial indices to speed up queries and evaluate a set of spatial indexing strategies.

- We define two sets of optimization rules to accelerate pipeline lineage queries.

- We present and analyze three real use cases of using finegrained data lineage for code validation, results inspection, and data cleaning, respectively.

The rest of the paper is organized as following: We briefly review the KeystoneML framework, the semantics of fine-grained lineage query, and present three diagnostic use cases in Section 2. Section 3 presents the data model in Hippo. Section 4 defines the primitive mapping types between input and output datasets. Section 5 and Section 6 describe the design and implementation of Hippo, respectively. We present performance measurements in Section 7 and conceptually compare Hippo with existing lineage system in Section 8. Finally, we conclude and envision future work in Section 9. 


\section{BACKGROUND}

In this section, we first review KeystoneML to help understand the technical background of this work. Then we discuss the basic lineage query functions in the context of ML pipelines. To motivate Hippo, we present three representative use cases of using finegrained lineage for ML pipeline diagnostics.

\subsection{KeystoneML}

In principle, Hippo is general for distributed ML frameworks whose programming interface can be abstracted as transformers and estimators. We integrate Hippo with KeystoneML to show the effectiveness of the design. KeystoneML is an application framework designed for the implementation of robust large-scale machine learning pipelines. Built on the principles of declarative programming and modular design, KeystoneML provides a light-weight and elegant API that allows users to describe these pipelines as the composition of two types of operator-transformers, which perform data transformation, and estimators, which "learn" transformers based on training data. KeystoneML pipelines are fit and executed in parallel using Apache Spark. These pipelines are compiled into an application directed acyclic graph (DAG) and optimized before execution. Current optimizations include online decisions about intermediate state materialization, standard optimizations such as common subexpression elimination, as well as machine-learning specific optimizations such as cost-based physical operator selection for solvers and other logical operators. KeystoneML includes a library of standard feature extractors in domains including computer vision, audio, and text processing, as well as standard statistical procedures and Estimators for several types of machine learning model.

Listing 1 shows a simplified image classification pipeline with KeystoneML. In the example, PixelScaler, GrayScaler, FeatureExtractor, and MatrixVectorizer are transformers while LeastSquareEstimator is an estimator. Applying this pipeline to the training dataset generates a new pipeline that consists of the first four transformers and an additional transformer with the linear model trained by LeastSquareEstimator. Line 8 shows how the trained pipeline is used for predictions with the test dataset.

Listing 1: A Simplified Image Classification Pipeline

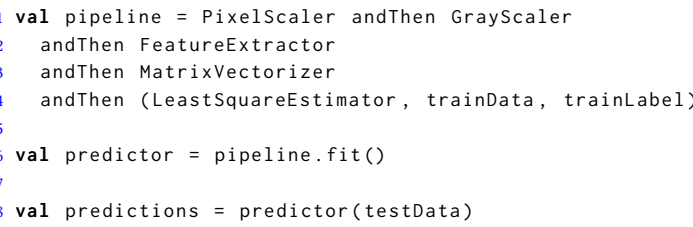

To define a transformer, users need to supply data transformation via the apply() interface. Listing 2 shows the PixelScaler example, where the transformation is to rescale an input image by dividing each pixel by 255.0 .

\section{Listing 2: PixelScaler Definition}

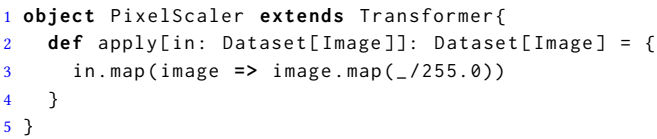

\begin{tabular}{|c|c|}
\hline \multicolumn{2}{|c|}{ GrayScaler } \\
\hline $\begin{array}{c}\text { Input } \\
\text { Coor }\end{array}$ & $\begin{array}{c}\text { Output } \\
\text { Coor }\end{array}$ \\
\hline$(0,0,0)$ & $(0,0)$ \\
\hline$(0,0,1)$ & $(0,0)$ \\
\hline$(0,0,2)$ & $(0,0)$ \\
\hline$(0,1,0)$ & $(0,1)$ \\
\hline$\cdots$ & $\cdots$ \\
\hline
\end{tabular}

\begin{tabular}{|c|c|}
\hline \multicolumn{2}{|c|}{ FeatureExtractor } \\
\hline $\begin{array}{c}\text { Input } \\
\text { Coor }\end{array}$ & $\begin{array}{c}\text { Output } \\
\text { Coor }\end{array}$ \\
\hline$(0,0)$ & $(0,0),(1,0), \ldots,(127,0)$ \\
\hline$(0,1)$ & $(0,0),(1,0), \ldots,(127,0)$ \\
\hline$(0,2)$ & $(0,1),(1,1), \ldots,(127,1)$ \\
\hline$(0,3)$ & $(0,1),(1,1), \ldots,(127,1)$ \\
\hline$\ldots$ & $\ldots$ \\
\hline
\end{tabular}

Figure 1: Cell Level Mapping in PixelScaler, GrayScaler, and FeatureExtractor.

\section{$2.2 \quad$ Fine-grained Lineage Query}

Conceptually, the fine-grained lineage of the pipeline shown in Listing 1 records the input and output cell mapping in a table for each transformer. Figure 1 shows the mapping tables of PixelScaler, GrayScaler, and FeatureExtractor. PixelScaler first scales the pixels of the 3-channel images with a factor of 255.0. Then GrayScaler converts the scaled images to single channel gray images. FeatureExtractor extracts visual features from the gray images then for each feature, it outputs a 128-dimension vector. For a single transformer such as PixelScaler, to trace the resulting output cells of an input cell, it filters the mapping table to find out records whose "Input Coor" fields are equal to the input cell. With multiple transformers in a chain, the fine-grained lineage is derived by joining a sequence of mapping tables on the "Output Coor" field of the current table and the "Input Coor" field of the next table. Then tracing the resulting output cells can be done in a similar way as in a single transformer.

Two typical tracing functions that a fine-grained lineage system provides are forward query and backward query. Forward query takes input coordinates as keys and returns the resulting output cells. Backward query takes output coordinates as keys and returns the contributing input cells. In the context of this paper, these queries return cell coordinates, and the actual cell value can be retrieved by accessing the data structure with the coordinates.

\subsection{Use Cases}

We now present three use cases which come from real ML pipelines of image classification and astronomy image processing. These three cases are representative of code validation, results inspection, and data cleaning, respectively, and demonstrate the utility of a fine-grained lineage system in the context of real world ML pipeline development.

2.3.1 Code Validation. In this case we use lineage for the debugging process as pipelines change and are augmented. Typical ML pipelines are constructed via an iterative process of refinement with a machine learning developer or data scientist in the loop. These users are constantly engineering new features, adding new datasources, and trying out new machine learning methods at all stages of the pipeline. Lineage offers a natural way for these users to hone in on the areas where two similar pipelines diverge in terms of their intermediate data, and presents a new avenue for 
investigation of model performance by allowing users to pinpoint the point at which their data diverged from a known good pipeline and to inspect what changed during the data processing procedure.

As a concrete example, consider the SIFTFisher pipeline, shown as Figure 2. It has been shown that augmenting traditional SIFT [23] (Scale-invariant feature transform) descriptors with their locations in the input image can improve classification performance. However, when we first developed such a pipeline, our results conflicted with published work indicating that these features provided a statistically significant improvement in classification accuracy. By employing lineage based debugging, we could isolate exactly where in the pipeline our calculations diverged from the original features, and diagnose impacts seen downstream like an under-fit GMM (Gaussian Mixture Model), and trace the ultimate lack of classification improvement to a bug in the underlying $\mathrm{C}$ library which we called into. Practically, this is done by first comparing the lineages of two executions to locate the intermediate result divergence in the SIFTExtractor transformer. Then by tracing the input of GMM backward to the output of SIFTExtractor, we could see that the new features added to our SIFT descriptors did not correspond to positions in the image. Rather they contained values outside of a suitable range for these features, which turned out to be randomly allocated memory. The SIFT feature investigation involves a backward query on the DimensionReducer and ColumnSampler transformer that applies Principal Component Analysis (PCA) and random sampling on the available vectors, respectively.

2.3.2 Results Inspection. Fine-grained data lineage of ML pipelines enables results interpretation, potential data anomaly detection, and retrospection of supporting input data.

One such use case is results inspection in the SourceExtractor pipeline, shown in Figure 3, that processes telescope images and produces astronomical object catalogs. The astronomical object catalog includes the position, shape, and other statistical properties of the object. In this case, astronomers find the abnormally bright objects over a threshold and validate that these objects are indeed astronomical objects rather than errors caused by malfunctioning CCD pixels. With the lineage information, astronomers can first filter the results to find the objects whose brightness is above the threshold. Then using backward query, they can find corresponding pixels for these bright objects. Either through mathematical analysis or human intervention, they are able to validate the correctness of the bright objects. Then the astronomer can use forward query to figure out how a bad pixel propagates to the catalogs. The backward query for this use case first joins with output data to find out the coordinates of the values that are over the threshold. Then the backward query is executed on the complete pipeline lineage to find the contributing pixels. If an erroneous pixel is confirmed, the forward query takes the erroneous pixel coordinate as the key and traces the complete pipeline lineage then return all results that are polluted by this erroneous pixel.

2.3.3 Data Cleaning. Another use of the lineage information is for users to apply a subset of the prepared dataset to fit the model. The ML expert who is building a SIFTFisher pipeline, shown in Figure 2, identifies bad training data items (e.g., the images that have both dogs and cats) due to measurement error. A natural investigation is then to remove these images, and test if that improves prediction accuracy.

With Hippo, the user can intercept the pipeline by loading the output of RowNormalizer (the last transformer before training process) directly into KeystoneML, filter out the data items that are derived from the images with both dogs and cats, and apply the filtered dataset directly to the linear solver. In this way, the rerunning process can bypass the expensive data preparation procedure (from PixelScaler to RowNormalizer) resulting in shorter turnaround time. Without lineage information, the user has to remove the according images from the training set and rerun the data preparation part (from PixelScaler to RowNormalizer), then feed the dataset to linear solver to train the model again.

\section{DATA MODEL}

Before discussing how ML pipeline cell-level dependency is recorded, we first introduce Hippo's data model and explain how to use this hierarchical model to represent the datasets in pipelines.

A dataset is often in the form of a nested data collection, e.g., Collection[Matrix]. Hippo uses a 3tier model to describe this nested data structure, as shown in Figure 4. On the top of the hierarchy is the "space" abstraction, which is defined by a number of dimensions. Each dimension corresponds to one layer of the nested collection. The cell positions inside the dataset are represented by the "coordinate" abstraction. In the middle of the model is the "shape" abstraction. The "shape" abstraction is a high-order representation of the underlying coordinates. Hippo describes a single coordinate as a point shape and a set of coordinates as a geometry shape, such as, a circle, a rectangle, or an ellipse. A space is then in the form of the union of a set of shapes. The "shape" abstraction is particularly space-efficient when storing lineage information in geometry mapping, which is discussed in details in Section 4.2

Taking an example dataset in the type of Collection[Matrix], Hippo analyzes the nested collection from outer to inner layer. In this case, the dataset is a $3 \mathrm{D}$ space where the first dimension corresponds to the outer collection, while the second and third dimension refer to the row and column of the matrix, respectively. The size of each dimension can be determined by the actual data structure. Please note that the Collection type in the model can be either a single-node collection such as a list or a distributed collection such as Resilient Distributed Dataset (RDD) in Apache Spark.

More formally, given a dataset in a nested collection, we refer to its outermost layer as Dimension 0, and its second outermost layer as Dimension 1, and so on. 


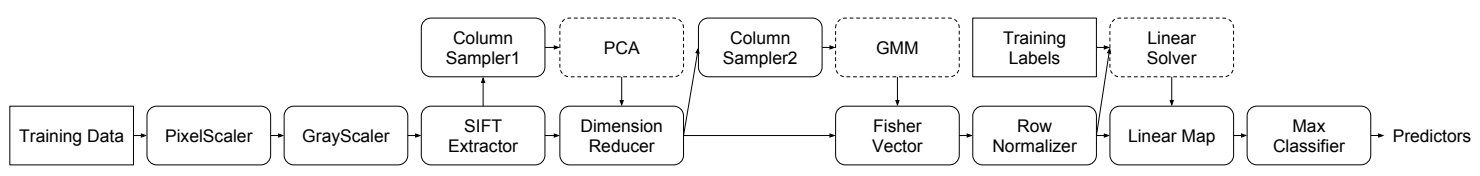

Figure 2: The SIFTFisher pipeline, boxes are dataset, rounded-corner boxes are transformers, dashed rounded-corner boxes are estimators.

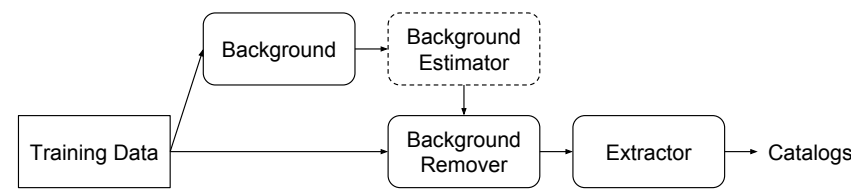

Figure 3: The SourceExtractor pipeline, boxes are dataset, rounded-corner boxes are transformers, dashed roundedcorner boxes are estimators.

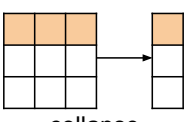

collapse

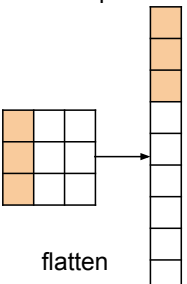

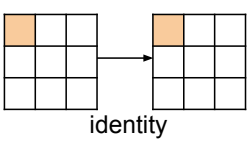

identity

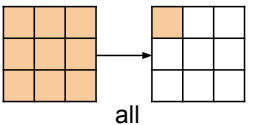

all

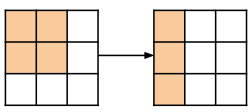

geometry

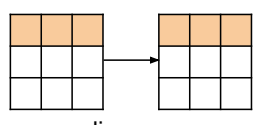

lincom

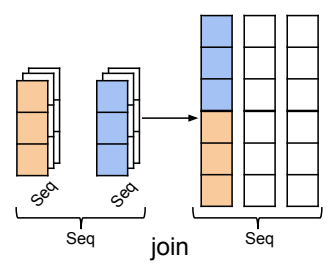

Figure 5: Primitive Mapping Types

\section{MAPPING TYPES}

At the core of Hippo is the cell-level dependency mappings between input and output datasets. In Section 3, we explain how to represent a dataset with a multi-dimensional space. In this section, we introduce the seven primitive mapping types to record the cell-level dependency between datasets.

\subsection{Overview}

We identify seven frequently occurring mapping types by analyzing the pre-defined data transformations of the KeystoneML, TensorFlow, and SystemML frameworks, From a higher dimension space to a lower dimension space, the mapping can be collapse or flatten. Between the equal dimension spaces, the mapping can be identity, all, geometry, lincom, and join. Figure 5 visualizes the seven primitive mapping types, with the involved cells highlighted.

\subsection{Geometry Mapping}

Geometry mapping records a many to many relation between input and output cells. Such data transformations take a group of cells from the input dataset and outputs another group of cells. These cell groups may be fit to higher order functions (geometries) such as circles, ellipses, and rectangles in a 2D space. Recording the mappings between higher order functions can significantly reduce the space consumption compared to the cell-wise mapping. In practice, a SIFT feature (a vector) can be recorded as a rectangle in a $2 \mathrm{D}$ space and its contributing pixels can be recorded as a circle of cells. Similarly for SourceExtractor, the light source pixels are in the shape of an ellipse and the output cells form a rectangle (a row) in a matrix. We also use the Geometry mapping for the column sampling operations, where a subset of input vectors is selected as output. The mapping is in the form of a pair of rectangles.

\subsection{Join Mapping}

Join Mapping is a fundamental mapping type that occurs any place two datasets are merged into one. We explore two common scenarios in machine learning processing pipelines. The first scenario is feature vector concatenation, where features of the same entity are extracted from different data sources. For example, a feature vector for an astronomical object in SourceExtractor may contain the image pixel values, the weather condition, and the camera condition. Data from these sources are processed separately and joined to a single vector with the object identity as the key. The other scenario is "labeling" in supervised learning, where users join the feature vectors with labels then feed them to the training process to obtain a model.

\subsection{Other Mappings}

Collapse mapping describes a space collapsing into a lower space along dimensions which need to be specified by the user. Examples include gray scaling a multi-channel image. Flatten mapping is where a space flattens to a one-dimension lower space, e.g., flattening a matrix to a vector with column major. For data transformations with a reverse direction as flatten mapping, e.g., reorganizing a vector into a matrix, users can still declare such mapping as flatten. Hippo can infer the mapping direction based on the input and output space.

In identity mapping, each output cell only depends on the cell with the identical coordinate in the input data structure, e.g., type conversion. We also extend the identity mapping to address the transpose operation with a user option to specify the two transposing dimensions. In all mapping, each output cell depends on all input cells. Lincom mapping describes the linear dependency in a data structure, e.g., vector normalization and linear model prediction.

\subsection{Mapping Coverage}

To verify the generality of these seven primitive mapping types, we analyze the data transformation operators in three distributed ML frameworks. These ML frameworks are KeystoneML v0.2.0, 
TensorFlow v0.7, and SystemML v0.9. They have 45, 146, and 81 predefined operators, respectively. Table 2 shows the operator coverage in these frameworks by each of the primitive mapping types.

Table 2: Hippo's Primitive Mapping Coverage in Existing Systems

\begin{tabular}{|c|c|c|c|c|c|c|c|c|}
\hline & Coll & Flat & Iden & All & Lincom & Join & Geo & Total \\
\hline \hline KeystoneML & $4 \%$ & $4 \%$ & $24 \%$ & $24 \%$ & $9 \%$ & $2 \%$ & $18 \%$ & $87 \%$ \\
\hline TensorFlow & $14 \%$ & $3 \%$ & $27 \%$ & $17 \%$ & $10 \%$ & $0 \%$ & $18 \%$ & $89 \%$ \\
\hline SystemML & $1 \%$ & $4 \%$ & $28 \%$ & $26 \%$ & $26 \%$ & $0 \%$ & $4 \%$ & $89 \%$ \\
\hline
\end{tabular}

In general, Hippo's primitive mapping types cover 90\% of each framework. The reason for the low presence of join mapping in TensorFlow and SystemML is that these two frameworks do not explicitly expose the combination of labels and feature vectors as a single operator. However, the join mapping occurs in every training process in supervised learning. For the rest of the mapping types that Hippo does not cover, we supply an additional CustomMapping() type, so users can use arbitrary data structure to record the cell-level dependency.

\section{DESIGN}

In Section 3 and 4, we present the underlying data model of Hippo and the primitive mapping types that record the cell-level dependency between the input and output datasets in a data transformation. In this section, we introduce the design of Hippo. We begin the discussion with the functional requirements of an ML pipeline diagnostic tool. Then we discuss how Hippo exposes its lineage declaration, instrumentation, and query interface to meet these requirements.

\subsection{Functional Requirements}

Hippo, as an ML pipeline diagnostic tool, has two basic functions: lineage capturing and serving. Lineage capturing requires a userfriendly, flexible, and powerful interface for lineage declaration and instrumentation. Lineage serving should be able to answer cell-level queries to enable diagnostics.

\subsection{Overview}

To study the applicability, we integrate Hippo with KeystoneML. Figure 6 shows the overview of Hippo and its interactions with other components. Rounded-corner rectangles are components around and inside KeystoneML. The two-sided arrows indicate interactions between components. Users compose machine learning pipelines with KeystoneML and submit the compiled DAG to Spark. All computation and I/O are done through the Spark Resilient Distributed Dataset (RDD) abstraction. With Hippo, users can declare and instrument lineage at the transformer level, so that the compiled DAG also contains lineage capturing and instrumentation operations. Lineage in Hippo is stored to HDFS [34] via Spark's RDD abstraction. Hippo can load the stored lineage in HDFS to Spark interactive command line interface. By interacting with the query interface, users can query cells of interest, replay transformation, or analyze the lineage for other purposes.

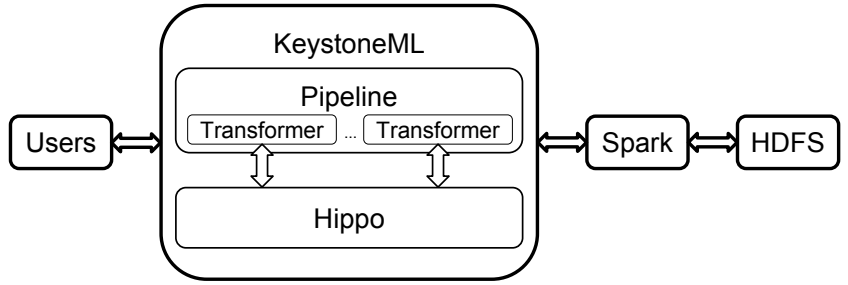

Figure 6: Overview of Hippo and its surrounding components.

\subsection{Lineage Interface}

Hippo exposes the lineage capturing interface by naming the lineage type with its mapping types between input and output datasets. All the type specific class inherits an abstract class of Lineage, which define common methods shared among the type specific classes:

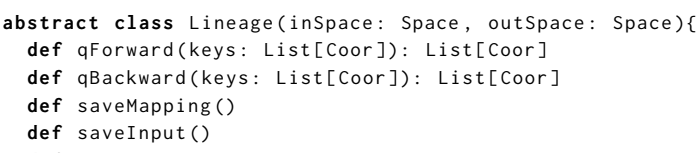

Listing 3 summarizes the lineage types and parameters. All these lineage classes inherent the abstract Lineage class.

\section{Listing 3: Hippo's Lineage Declaration Interface}

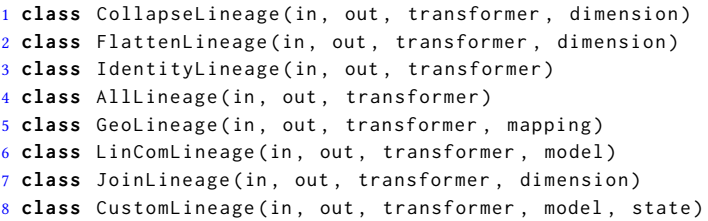

In general, Hippo collects six types of data: the input dataset (in), the output dataset (out), the transformer (transformer), the customized mapping (mapping), the optional model (model), and one state parameter which captures the internal state of the transformer, e.g., a random seed in a column sampler.

Hippo's lineage declaration interface can determine the space, the dimension count, and the size of each dimension of the datasets by examining the nested data structure and the parameters specified by users.

\section{Listing 4: PixelScaler with Lineage Definition}

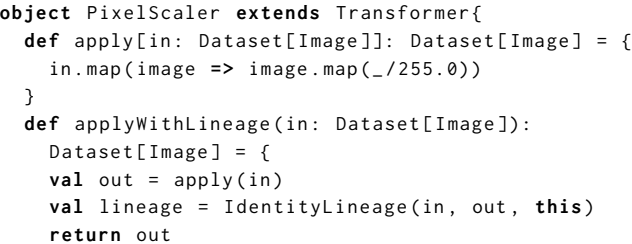

For example, Listing 4 rewrites the PixelScaler transformer in Listing 2 with lineage declaration. Line 8 in the listing instantiates the spaces with the input and output datasets, and specify the mapping type as Identity. 


\section{IMPLEMENTATION}

There are three performance requirements for an ML pipeline diagnostic tool. First, the lineage capturing should add a modest wall clock time overhead to the original pipeline. Second, the storage space of the lineage should be within a manageable size. Third, the lineage serving function should be with a low latency for interactive diagnostics. As documented in the report [27] in human computer interaction, 1 second latency is the boundary to keep users' thought flow while 10 seconds is the boundary to loose users' attention.

In this section, we present system implementation details to address the three performance requirements.

\subsection{Metadata Separation}

Hippo separates the metadata of a data structure (e.g., the coordinates of cells in a matrix) from the actual data and leaves it as an option for users to decide whether or not to collect the actual data.

Under the constraint of complete pipeline lineage integrity, a minimal lineage to be captured is all the metadata mapping of the transformers. All the intermediate and result datasets can always be reproduced with the KeystoneML transformer given the its deterministic replay property. A profile on the SIFTFisher and SourceExtractor pipeline shows that this metadata separation can reduce storage space by a factor of $13.7 \mathrm{x}$ (from 725.6 GB to 109.9 GB) and $2480.9 \mathrm{x}$ (from $507.1 \mathrm{G}$ to $204.4 \mathrm{M}$ ), respectively.

\subsection{Lineage Storage}

Hippo uses the RDD abstraction for lineage information storage. At a high level, Hippo creates a unique directory on the underlying file system with a naming pattern of concatenating the transformer name and the initial input dataset identifier of the pipeline. In this way, Hippo can tell between the training path and testing path based on the input dataset identifier, if the training and testing are run in a single program with distinct datasets.

In the current implementation, Hippo stores lineage information as serialized objects in HDFS. However, this lineage information could just as easily be stored virtually on any distributed storage system, including an in-memory system such as Alluxio [21].

\subsection{Geometry Mapping Indexing}

Among the primitive mapping types, geometry mapping consumes the most space due to its lack of a regular mapping pattern. In a geometry mapping, the geometries can be randomly spread in a space and the pairing between input and output geometries can also be random. Using high-order functions (the shape abstraction in the data model) can reduce the storage space, but querying the cells in the high-order functions can be slow due to the encoding. In this section, we present four indexing strategy to address the slow querying problem. The four indexing strategies are referred to as NoIndex, Direct, RTree, and KMeans, respectively. An empirical performance study is presented in Section 7.1. For simplicity, only forward query is discussed in this section. The backward query works in a similar way by reverting the geometry pair.

Figure 7 shows the mapping example in the following discussion. Table 3 shows the $2 \mathrm{D}$ geometry mapping.
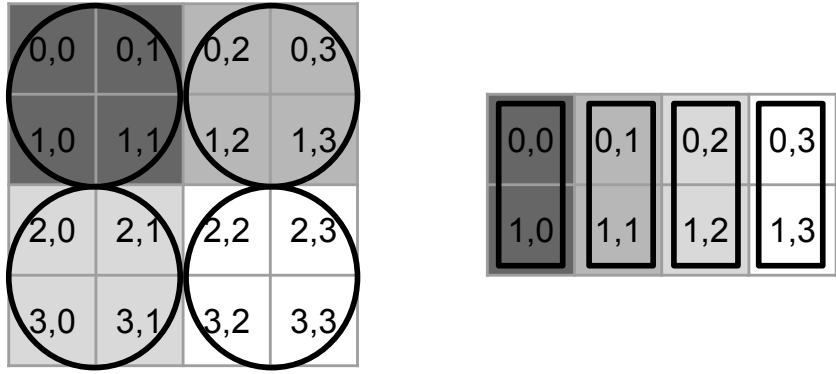

Figure 7: A geometry mapping example, the left matrix is input and the right matrix is output. The mapping between the geometries are shown using gray shades.

Table 3: Cell-wise and geometry mapping of the example in Figure 7

\begin{tabular}{|c|c|c|c|}
\hline Input & Geometry & Output & Geometry \\
\hline \hline$(0,0), \ldots,(1,1)$ & Circle_0 & $(0,0),(1,0)$ & Rect_0 \\
\hline$(0,2), \ldots,(1,3)$ & Circle_1 & $(0,1),(1,1)$ & Rect_1 \\
\hline$(2,0), \ldots,(3,1)$ & Circle_2 & $(0,2),(1,2)$ & Rect_2 \\
\hline$(2,2), \ldots,(3,3)$ & Circle_3 & $(0,3),(1,3)$ & Rect_3 \\
\hline
\end{tabular}

6.3.1 No Index. Using the example in Table 3, with NoIndex strategy, a query for a cell needs to traverse all circles (input geometries). If the cell is in a circle, the query returns all output cells expanded from the mapping rectangle.

6.3.2 Direct Index. To build the Direct index, Hippo uses each input cell as key and the associated geometry as the value, as shown in Figure 8. Hippo relies on the underlying runtime system for the optimization of storing repeated geometries with a single copy. A query on a cell directly returns the cells in the corresponding rectangle.

6.3.3 RTree Index. R-tree [18] indices are widely used to index multidimensional information. Variants of R-tree, such as R+-tree [33] and $\mathrm{R}^{*}$ tree [5], seek to improve the worst case query by minimizing the overlapped geometry at leaf nodes by paying the index building cost. In our use cases, it is rare that a single cell occurs in all geometries. Thus R-tree is sufficient to validate the idea of using spatial indexing for geometry mapping. Hippo uses the Archery [30] package to build the RTree index.

6.3.4 KMeans Index. Since the geometries are spread in a space, it is reasonable to first cluster these geometries then build a hierarchical spatial index on the clusters. We design a two-layer tree index with its leaf nodes as a cluster of geometries and its non-leaf nodes as the bounding box of the cluster. The geometries are distributed to each cluster using the KMeans [24] algorithm. 


\subsection{Key Count and Mapping Sequence Reduction}

With Hippo, the lineage of an ML pipeline is in the form of a sequence of mappings, with each mapping recording the cell-level dependency for each transformer. Querying one cell in the very beginning mapping can return all resulting output cells of the last transformer. In this process, Hippo recursively query the mappings in the sequence with the previous results as keys. However, the number of intermediate keys can explode depending on the transformer. For example, FisherVector transforms a matrix of 80 rows and 60,000 columns to a matrix of 80 rows and 512 columns in an all mapping type. A backward query of any output cell will result in 4.8 million intermediate keys, which will be used to query the remaining mappings. In a naive sense, if the next mapping is also an all mapping, then there is an opportunity to reduce the 4.8 million queries to a single query as all these queries return the same result.

Based on this intuition, we define two types of optimizations to reduce key count. The first set of rules is to check the keys' totality in the input space. If the keys include every cell in the input space, then the query returns all cells in the output space. The only exception is geometry mapping, where it returns all cells in all output geometries. The second set of rules exploits the structure of collapse and lincom mapping. For example in collapse mapping along column direction, forward queries with cells in the same row will return identical results. Thus, Hippo will query only once with one of these cells.

One additional optimization is to reduce the number of mappings in a pipeline. For example, a forward query on an identity mapping followed by a collapse mapping has the exact same results as querying solely on the collapse mapping. Thus we can reduce the two mappings to a single one. The complete list of reduction rules is:

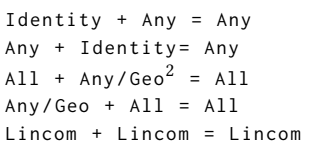

If a pair of mappings can not be reduced, Hippo simply records them as a sequence of mappings.

\section{EVALUATION}

In this section, we evaluate a number of aspects of Hippo's performance. For geometry mapping indexing strategies, we measure the build time, query performance, memory usage and storage consumption. Then we present Hippo's lineage capturing wall clock time overhead with two real ML pipelines on varying cluster sizes. We also measure the performance of the three use cases presented in Section 2.3.

All experiments are run on Amazon EC2 using r3.8xlarge instances. Each machine has 16 physical cores, 244 GB memory, and two 320GB SSDs. Hippo is integrated with KeystoneML 0.3 which in turn runs over Spark 1.3.1 and HDFS from the CDH4 distribution of Hadoop.

The two pipelines we are using are SIFTFisher and SourceExtractor. The datasets are VOC2007 [12] and a subset of Sloan Digital

\footnotetext{
${ }^{2}$ Geo Mapping is not included in Rule 3 and Rule 4
}

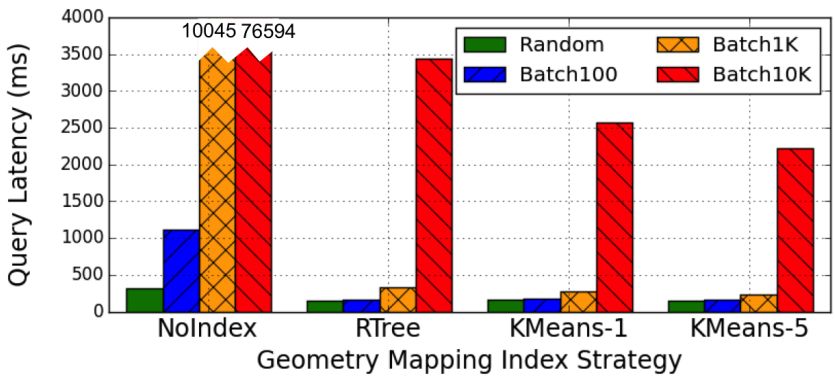

Figure 9: Query performance profile of the geometry mapping with four indexing strategies

Table 4: Summary of Build Time and Additional Memory Usage (over 0.7 TB) for the Four Indexing Strategies

\begin{tabular}{|c|c|c|}
\hline Index & Build Time & Additional Mem \\
\hline \hline NoIndex & $0 \mathrm{~s}$ & $71.4 \%$ \\
\hline RTree & $30.6 \pm 1.5 \mathrm{~s}$ & $85.7 \%$ \\
\hline KMeans- 1 & $66.6 \pm 4.2 \mathrm{~s}$ & $85.7 \%$ \\
\hline KMeans-5 & $342.3 \pm 8 \mathrm{~s}$ & $114.3 \%$ \\
\hline
\end{tabular}

Sky Survey [43] (SDSS) Data Release 7, respectively. The VOC2007 dataset contains 10,000 images with a total size of $828 \mathrm{MB}$. The SDSS DR7 dataset contains 11,150 images with a total size of 65 GB.

\subsection{Geometry Mapping Index}

With this experiment we seek to profile the query latency, index build time, and memory usage of the indexing strategies for geometry mapping discussed in Section 6.3. In particular, KMeans-1 and KMeans -5 builds the index with one and five iterations, respectively. We use a workload derived from the SIFTExtractor transformer in the SIFTFisher pipeline. The mapping has 5,000 pairs of input and output matrix, with each pair producing 60,000 geometry tuples (300 million in total) that are uniformly distributed in a 2D space. The experiment is executed on a 16 machine cluster.

Figure 9 presents the query performance with these indexing strategies. For each strategy, we run queries with $\{1,100,1 \mathrm{~K}, 10 \mathrm{~K}\}$ keys. The Direct strategy crashes due to insufficient memory. NoIndex strategy shows a high query latency which quickly grows over 10,000 ms with 100 keys. The other three strategies show a similar scalability, while KMeans- 1 runs $17.9 \%$ and $25.1 \%$ faster than RTree with $1 \mathrm{~K}$ and $10 \mathrm{~K}$ keys, respectively. KMeans- 5 runs $31.1 \%$ and $35.2 \%$ faster than RTree with $1 \mathrm{~K}$ and $10 \mathrm{~K}$ keys, respectively.

Table 4 summarizes the index build time and additional memory usage. The size of the geometry mapping is $30.8 \mathrm{~GB}$. To summarize, NoIndex has the highest query latency and it is not practical for real use. KMeans- 5 has the best query performance and the longest index building time. RTree has a balanced query performance and building time. Thus Hippo uses the RTree strategy as default, leaving the other strategies as users' options.

\subsection{Lineage Capturing Overhead}

We evaluate lineage capturing wall clock time overhead with the SIFTFisher (test path) and SourceExtractor pipelines. For each pipeline, 
Table 5: Data Size of SIFTFisher and SourceExtractor with the Meta-only and Meta+Data settings

\begin{tabular}{|c|c|c|}
\hline Pipeline & SIFTFisher & SourceExtractor \\
\hline \hline Meta-only & $109.9 \mathrm{~GB}$ & $204.4 \mathrm{MB}$ \\
\hline Meta+Data & $615.7 \mathrm{~GB}$ & $506.9 \mathrm{~GB}$ \\
\hline
\end{tabular}

Table 6: Relative Lineage Capturing Wall Clock Time Overhead

\begin{tabular}{|c|c|c|c|}
\hline Pipeline \& Setting & 8 nodes & 16 nodes & 32 nodes \\
\hline \hline SIFTFisher Meta-only & $38.4 \% \pm 2.6 \%$ & $34.3 \% \pm 0.9 \%$ & $30.4 \% \pm 0.6 \%$ \\
\hline SIFTFisher Meta+Data & $142.7 \% \pm 3.7 \%$ & $88 \% \pm 1.9 \%$ & $60.6 \% \pm 0.8 \%$ \\
\hline SE Meta-only & $39.3 \% \pm 2.9 \%$ & $23.4 \% \pm 0.9 \%$ & $20.7 \% \pm 0.6 \%$ \\
\hline SE Meta+Data & $238.5 \% \pm 11.2 \%$ & $94.4 \% \pm 1.3 \%$ & $54 \% \pm 0.7 \%$ \\
\hline
\end{tabular}

we measure the wall clock time of lineage capturing with two settings. With the first setting (meta-only), Hippo only captures the metadata mapping, since this is the minimal lineage information to preserve the pipeline lineage integrity. With the second setting (meta+data), Hippo captures both the metadata mapping and the output dataset of each transformer. With the lineage information captured in this way, queries on the output dataset does not require any rerunning of data transformations. This is considered as the minimal sufficient lineage information of a pipeline without rerunning. Table 5 presents the dataset size collected with both settings for both pipelines.

Table 6 summarizes the relative wall clock time overhead of lineage capturing of the SIFTFisher and SourceExtractor pipeline. Across the scales, the meta-only lineage capturing introduces a $30 \%-40 \%$ percent overhead compared to the lineage-free SIFTFisher pipeline. The SourceExtractor pipeline's meta-only capturing overhead is $20 \%-40 \%$. Though capturing all the intermediate data introduces additional overhead for both pipelines, the overhead decreases near linearly with more machines. This is because the additional intermediate data collection is simply writing more data to HDFS and these writing operations scale with more machines.

A further overhead decomposition of the 16 node measurements reveals that the SIFTExtractor transformer contributes to $85.4 \%$ of the lineage capturing overhead. The individual overhead for the rest of the transformers ranges from $1.1 \%$ to $1.4 \%$. This is because we use Geometry mapping for SIFTExtractor and the Geometry mapping is the only mapping type that materializes cell-level dependency at runtime among the primitive types. Similarly, the Extractor transformer in the SourceExtractor pipeline solely contributes $65.2 \%$ to the lineage capturing overhead.

\subsection{Query Latency and Scalability}

In Section 6.4, we stated that in the case of a pipeline lineage query, the intermediate key number can increase dramatically. The query with this large key number on a single mapping can impact the query performance substantially. This performance measurement seeks to profile the query scalability of Hippo on a single mapping with a varying key number.

We run queries with varying key numbers and present the performance in Figure 10. For each lineage type, we use a collection of 5,000 pairs of input and output data structures (vector, matrix,

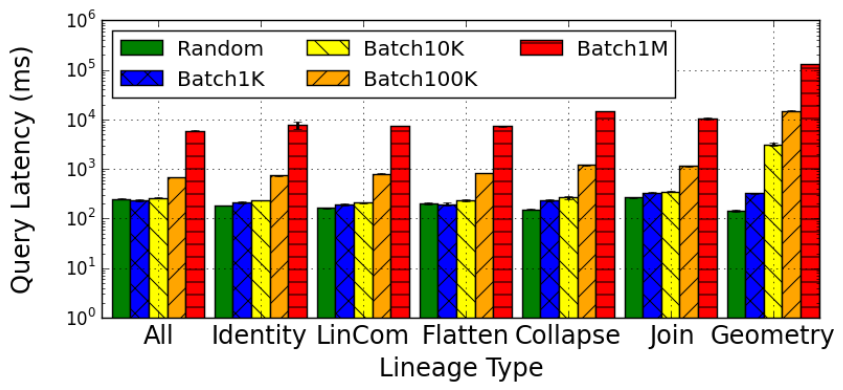

Figure 10: Query latency for the seven lineage types (in $\log$ scale).

Table 7: Data Structure Settings

\begin{tabular}{|c|c|c|}
\hline Type & Input Structure & Output Structure \\
\hline \hline All & Vector $(40960)$ & Vector $(40960)$ \\
\hline Identity & Matrix $(80,512)$ & Matrix $(80,512)$ \\
\hline LinCom & Vector $(40960)$ & Vector $(20)$ \\
\hline Flatten & Matrix $(80,512)$ & Vector $(40960)$ \\
\hline Collapse & Image $(375,500,3)$ & Matrix $(375,500)$ \\
\hline Join & Matrix $(500,300)$, Matrix $(500,300)$ & Matrix $(1000,300)$ \\
\hline & Matrix $(300,500)$, & Matrix $(128,60000)$, \\
Geometry & $60 \mathrm{~K}$ geometries & $60 \mathrm{~K}$ geometries \\
\hline
\end{tabular}

image) and distribute them over 16 machines. Table 7 shows the metadata settings of the input and output data structure in each query

Given the fact that all mapping, identity mapping, lincom mapping, flatten mapping, collapse mapping, and join mapping only involve trivial computation, queries with 1 to $10 \mathrm{~K}$ keys show latency under $200 \mathrm{~ms}$. These queries show performance degradation with $100 \mathrm{~K}$ keys (around 1 second). From $100 \mathrm{~K}$ keys, the query latency increases proportionally with the key number as shown with the $1 \mathrm{M}$ key performance. Geometry Mapping shows a consistent latency under $200 \mathrm{~ms}$ from 1 to $1 \mathrm{~K}$ keys. Beyond that, a query with $10 \mathrm{~K}$ keys takes $2.41 \pm 0.6$ seconds and a query with $1 \mathrm{M}$ keys take $132.9 \pm 0.4$ seconds

This query scalability profile shows low latency $(\sim 200 \mathrm{~ms})$ for single cell query in a mapping. With the end-to-end pipeline lineage, the query can be slow due to the quickly increasing key number. While in the case of SIFTFisher pipeline, it can take an estimated $O\left(10^{3}\right)$ seconds to finish as the backward query on the SIFTExtractor transformer with a key count of $7.7 \mathrm{M}$. The actual mapping is from a matrix with 128 rows and $60 \mathrm{~K}$ columns to a matrix with 333 rows and 500 columns. With the optimization rules in Section 6.4, we are able to query the lineage of the complete SIFTFisher pipeline (11 transformers in total) in $75.7 \pm 1.7$ seconds.

\subsection{Use Cases}

In the code validation case, users instrument the GMM training path of the SIFTFisher pipeline to collect both the metadata mapping and intermediate data. As shown in Figure 2, the GMM training path omits PCA and ColumnSampler1. Upon validation, users load the lineage of DimensionReducer and ColumnSampler2 then apply a backward query on one result vector. With the result coordinates, 
the users access the actual values of the contributing vector and find out that the new features contain values outside of the suitable range as pixel positions in an image. Such an instrumentation introduces a wall clock time overhead of $60.9 \%$ on a 16 machine cluster. The backward query and value retrieval takes $0.44 \pm 0.01$ seconds.

In the results inspection use case, the user of the SourceExtractor pipeline captures the metadata mapping in all transformers along with the output datasets of the Extractor transformer. By joining the metadata mapping with the output dataset, which contains the luminosity information, he first filters out the objects with a threshold brightness. Then the user queries the metadata mapping to find the positions of corresponding pixels in the input images. This instrumentation introduces a wall clock time overhead of $40.8 \%$ on a 8 machine cluster. Filtering luminous flux $>300,000$ lumen over 1 million objects returns 10,283 objects with the corresponding pixel positions in $1.84 \pm 0.14$ seconds. A forward query that traces the error propagation given a bad pixel takes $3.3 \pm 0.03$ seconds to return.

In the data cleaning use case, the user of the SIFTFisher pipeline wants to remove the training images that have both dogs and cats. So he instruments the pipeline to capture all metadata mappings and the output datasets for NormalizeRows transformer. Upon removal, he first joins the output dataset of NormalizeRows (a RDD of vectors) with the labels. Then he filters out the corresponding vectors with both cats and dogs in it ( eight vectors corresponding to eight images in this case). From here, he can try retrain the model with the cleaned dataset. This particular instrumentation introduces a wall clock time overhead of $46.8 \%$, and the removal takes $25.7 \pm 0.27$ seconds. Compared to the case of rerunning the data preparation phase of the pipeline, using lineage information speeds up the turnaround time by $16.4 \mathrm{x}$ (from $421.3 \pm 3.8$ seconds to $25.7 \pm 0.3$ seconds).

\section{RELATED WORK}

Coarse-grained Lineage: Researchers have extensively studied lineage (in some cases referred as provenance) in different contexts. Scientific workflow systems such as Chimera [13], Taverna [28], ESSW [15], Kepler [2], and Karma2 [36] collect coarse-grained lineage of files and computation. Similar work has been well summarized by surveys $[6,14,35]$. Recent work by Altintas [1] investigates how to integrate workflow lineage in a collaborative environment. Coarse-grained lineage is useful in these systems to trace the data and code dependencies. However, it lacks the cell-level details for ML pipelines diagnosis.

Fine-grained Lineage: Fine-grained lineage has been investigated in the context of data visualization [39, 41], data warehouse [9, 10], RDBMS [40], and user-curated database [8]. Recent works of RAMP [19, 31] and Newt [22] capture fine-grained lineage for the MapReduce systems. The Lipstick [3] system collects fine-grained lineage on declarative querying language Pig Latin [29]. SubZero [42] proposes a lineage system for the arraybased SciDB [7]. Titian [20] enables fine-grained lineage capturing and query for Apache Spark. All these systems design lineage capturing interface on the well-defined low level operators. For example, the data warehouse has the aggregate, select, project, join operators. RDBMS has its SQL operators. MapReduce systems formulates the computation as mappers and reducers. The curated database defines four operators of insert, delete, copy and paste. Though lineage capturing interface designed in these systems is general to support all applications running on top them, they are not suitable for the ML pipeline diagnostics. This is because ML frameworks do not have such a set of well-defined low level operators.

Although Titian solves the lineage problem on Spark, it is insufficient for ML pipelines. This is because Titian tracks lineage at the RDD element level while ML pipeline lineage requires the cell-level mapping inside a RDD element. One of SubZero's contributions is the region lineage that collects fine-grained lineage for UDFs besides the built-in operators in SciDB. However, region lineage is not sufficient for ML pipelines because it only captures many to one relationships between input and output cells, while ML pipelines require many to many relationships as well. Besides, SubZero is implemented on a single computer while Hippo has to work in a distributed environment.

The Taverna 2 workflow system [38] proposes a fine-grained lineage model and the work by Missier et al. [26] presents two mapping types as data collection cross-product and index-projection. These mapping types partially address the workflow system computation mapping, however, they are not sufficient to cover the data transformation in ML pipelines.

Other Fine-grained Lineage Capturing Techniques: Researchers also have tried to collect fine-grained lineage with other approaches. Weak inversion and verification methods [41] are proposed to support approximate lineage collection. Dynamic program analysis is employed to capture fine-grained lineage inside nonrelational operators [45] with a 7.5 39.8x slowdown compared to the original program. These approaches do not fit the ML pipeline lineage scenario since they either lose the lineage accuracy or introduce excessive performance penalty. The Arnold system [11] can collect fine-grain lineage for operating system processes, however it loses track of the upper level data structures (e.g., vector, matrix, images), which are fundamental to ML pipelines.

\section{CONCLUSION AND FUTURE WORK}

Hippo enables interactive ML pipeline diagnosis such as code validation, results inspection, and data cleaning by leveraging finegrained data lineage. Hippo exposes an elegant and powerful interface for users to specify and instrument lineage capturing. This interface has a coverage of $\sim 90 \%$ of the transformers in the current KeystoneML, TensorFlow, and SystemML code base. Combining various techniques and optimizations, Hippo is able to reduce the lineage storage space by a factor of $O\left(10^{3}\right)$ compared to the baseline of cell-wise mapping recording and Hippo can answer typical queries within a few seconds to enable interactive ML pipeline diagnosis.

As future work, we are investigating the diagnosability of other model families such as the bayesian network. We will also explore the diagnosability inside the training process. We seek to provide users with fine-grained lineage for queries such as finding supporting training samples for a prediction and the impact of certain feature removal or training data item removal. 


\section{ACKNOWLEDGMENTS}

This research is supported in part by NSF CISE Expeditions Award CCF-1139158, LBNL Award 7076018, and DARPA XData Award FA8750-12-2-0331, and gifts from Amazon Web Services, Google, SAP, The Thomas and Stacey Siebel Foundation, Adatao, Adobe, Apple, Inc., Blue Goji, Bosch, C3Energy, Cisco, Cray, Cloudera, EMC, Ericsson, Facebook, Guavus, Huawei, Intel, Microsoft, NetApp, Pivotal, Samsung, Splunk, Virdata, VMware, and Yahoo!.

\section{REFERENCES}

[1] I. Altintas, M. K. Anand, D. Crawl, S. Bowers, A. Belloum, P. Missier, B. Ludäscher C. A. Goble, and P. M. Sloot. Understanding collaborative studies through interoperable workflow provenance. In Provenance and Annotation of Data and Processes, pages 42-58. Springer, 2010.

[2] I. Altintas, O. Barney, and E. Jaeger-Frank. Provenance collection support in the kepler scientific workflow system. In Provenance and annotation of data, pages 118-132. Springer, 2006

[3] Y. Amsterdamer, S. B. Davidson, D. Deutch, T. Milo, J. Stoyanovich, and V. Tannen. Putting lipstick on pig: Enabling database-style workflow provenance. Proceedings of the VLDB Endowment, 5(4):346-357, 2011.

[4] Apache. Apache Hadoop. http://hadoop.apache.org/.

[5] N. Beckmann, H.-P. Kriegel, R. Schneider, and B. Seeger. The R*-tree: An efficient and robust access method for points and rectangles. In Proceedings of the 1990 ACM SIGMOD International Conference on Management of Data, SIGMOD '90, pages 322-331, New York, NY, USA, 1990. ACM.

[6] R. Bose and J. Frew. Lineage retrieval for scientific data processing: a survey. ACM Computing Surveys (CSUR), 37(1):1-28, 2005.

[7] P. G. Brown. Overview of scidb: large scale array storage, processing and analysis. In Proceedings of the 2010 ACM SIGMOD International Conference on Management of data, pages 963-968. ACM, 2010.

[8] P. Buneman, A. Chapman, and J. Cheney. Provenance management in curated databases. In Proceedings of the 2006 ACM SIGMOD international conference on Management of data, pages 539-550. ACM, 2006.

[9] Y. Cui and J. Widom. Practical lineage tracing in data warehouses. In Data Engineering, 2000. Proceedings. 16th International Conference on, pages 367-378 IEEE, 2000

[10] Y. Cui and J. Widom. Lineage tracing for general data warehouse transformations. The VLDB fournal, The International fournal on Very Large Data Bases, 12(1):41-58, 2003.

11] D. Devecsery, M. Chow, X. Dou, J. Flinn, and P. M. Chen. Eidetic systems. In Proceedings of the 11th USENIX Symposium on Operating Systems Design and Implementation (OSDI), 2014.

[12] M. Everingham, L. Van Gool, C. K. I. Williams, J. Winn, and A. Zisserman. The PASCAL Visual Object Classes Challenge 2007 (VOC2007) Results. http://www.pascalnetwork.org/challenges/VOC/voc2007/workshop/index.html.

[13] I. Foster, J. Vöckler, M. Wilde, and Y. Zhao. Chimera: A virtual data system for representing, querying, and automating data derivation. In Scientific and Statistical Database Management, 2002. Proceedings. 14th International Conference on, pages 37-46. IEEE, 2002.

[14] J. Freire, D. Koop, E. Santos, and C. T. Silva. Provenance for computational tasks: A survey. Computing in Science \& Engineering, 10(3):11-21, 2008.

[15] J. Frew and R. Bose. Earth system science workbench: A data management infrastructure for earth science products. In Scientific and Statistical Database Management, 2001. SSDBM 2001. Proceedings. Thirteenth International Conference on, pages 180-189. IEEE, 2001.

[16] A. Ghoting, R. Krishnamurthy, E. Pednault, B. Reinwald, V. Sindhwani, S. Tatikonda, Y. Tian, and S. Vaithyanathan. Systemml: Declarative machine learning on mapreduce. In Data Engineering (ICDE), 2011 IEEE 27th International Conference on, pages 231-242. IEEE, 2011.

[17] Google. Tensorflow. http://tensorflow.org/.

[18] A. Guttman. R-trees: A dynamic index structure for spatial searching. In Proceedings of the 1984 ACM SIGMOD International Conference on Management of Data, SIGMOD '84, pages 47-57, New York, NY, USA, 1984. ACM.

[19] R. Ikeda, H. Park, and J. Widom. Provenance for generalized map and reduce workflows. In CIDR 2011. Stanford InfoLab.

[20] M. Interlandi, K. Shah, S. D. Tetali, M. A. Gulzar, S. Yoo, M. Kim, T. Millstein, and T. Condie. Titian: data provenance support in spark. Proceedings of the VLDB
Endowment, 9(3):216-227, 2015.

[21] H. Li, A. Ghodsi, M. Zaharia, S. Shenker, and I. Stoica. Tachyon: Reliable, memory speed storage for cluster computing frameworks. In Proceedings of the ACM Symposium on Cloud Computing, pages 1-15. ACM, 2014.

[22] D. Logothetis, S. De, and K. Yocum. Scalable lineage capture for debugging disc analytics. In Proceedings of the 4th annual Symposium on Cloud Computing, page 17. ACM, 2013.

[23] D. G. Lowe. Object recognition from local scale-invariant features. In Computer vision, 1999. The proceedings of the seventh IEEE international conference on, volume 2, pages 1150-1157. Ieee, 1999.

[24] J. MacQueen et al. Some methods for classification and analysis of multivariate observations. In Proceedings of the fifth Berkeley symposium on mathematical statistics and probability, volume 1, pages 281-297. Oakland, CA, USA., 1967.

[25] X. Meng, J. Bradley, B. Yavuz, E. Sparks, S. Venkataraman, D. Liu, J. Freeman, D. Tsai, M. Amde, S. Owen, et al. Mllib: Machine learning in apache spark. arXiv preprint arXiv:1505.06807, 2015.

[26] P. Missier, N. W. Paton, and K. Belhajjame. Fine-grained and efficient lineage querying of collection-based workflow provenance. In Proceedings of the 13th International Conference on Extending Database Technology, pages 299-310. ACM, 2010.

[27] J. Nielsen. Powers of 10: Time scales in user experience, 2009. http://www. nngroup.com/articles/powers-of-10-time-scales-in-ux/.

[28] T. Oinn, M. Greenwood, M. J. Addis, M. N. Alpdemir, J. Ferris, K. Glover, C. Goble, A. Goderis, D. Hull, D. Marvin, et al. Taverna: lessons in creating a workflow environment for the life sciences. Fournal of Concurrency and Computation: Practice and experience, 2002.

[29] C. Olston, B. Reed, U. Srivastava, R. Kumar, and A. Tomkins. Pig latin: a not-soforeign language for data processing. In Proceedings of the 2008 ACM SIGMOD international conference on Management of data, pages 1099-1110. ACM, 2008.

[30] E. Osheim. Archery. https://github.com/meetup/archery.

[31] H. Park, R. Ikeda, and J. Widom. RAMP: A system for capturing and tracing provenance in mapreduce workflows. In 37th International Conference on Very Large Data Bases (VLDB). Stanford InfoLab, August 2011.

[32] F. Pedregosa, G. Varoquaux, A. Gramfort, V. Michel, B. Thirion, O. Grisel, M. Blondel, P. Prettenhofer, R. Weiss, V. Dubourg, et al. Scikit-learn: Machine learning in python. The fournal of Machine Learning Research, 12:2825-2830, 2011.

[33] T. Sellis, N. Roussopoulos, and C. Faloutsos. The R+-tree: A dynamic index for multi-dimensional objects. 1987.

[34] K. Shvachko, H. Kuang, S. Radia, and R. Chansler. The Hadoop Distributed File System. In Proceedings of the IEEE Symposium on Mass Storage Systems and Technologies (MSST'10), pages 1-10. IEEE, 2010.

[35] Y. L. Simmhan, B. Plale, and D. Gannon. A survey of data provenance in e-science. ACM Sigmod Record, 34(3):31-36, 2005

[36] Y. L. Simmhan, B. Plale, and D. Gannon. Karma2: Provenance management for data-driven workflows. Web Services Research for Emerging Applications: Discoveries and Trends: Discoveries and Trends, page 317, 2010.

[37] E. Sparks, S. Venkataraman, T. Kaftan, M. Franklin, and B. Recht. Keystoneml. https://github.com/amplab/keystone.

[38] J. Sroka, J. Hidders, P. Missier, and C. Goble. A formal semantics for the taverna 2 workflow model. Journal of Computer and System Sciences, 76(6):490-508, 2010.

[39] M. Stonebraker, J. Chen, N. Nathan, C. Paxson, and J. Wu. Tioga: Providing data management support for scientific visualization applications. In VLDB, volume 93, pages 25-38. Citeseer, 1993.

[40] J. Widom. Trio: A system for integrated management of data, accuracy, and lineage. Technical Report 2004-40, Stanford InfoLab, August 2004.

[41] A. Woodruff and M. Stonebraker. Supporting fine-grained data lineage in a database visualization environment. In Data Engineering, 1997. Proceedings. 13th International Conference on, pages 91-102. IEEE, 1997.

[42] E. Wu, S. Madden, and M. Stonebraker. SubZero: A fine-grained lineage system for scientific databases. In Data Engineering (ICDE), 2013 IEEE 29th International Conference on, pages 865-876. IEEE, 2013.

[43] D. G. York, J. Adelman, J. E. Anderson Jr, S. F. Anderson, J. Annis, N. A. Bahcall, J. Bakken, R. Barkhouser, S. Bastian, E. Berman, et al. The sloan digital sky survey: Technical summary. The Astronomical fournal, 120(3):1579, 2000.

[44] M. Zaharia, M. Chowdhury, T. Das, A. Dave, J. Ma, M. McCauley, M. Franklin, S. Shenker, and I. Stoica. Resilient distributed datasets: A fault-tolerant abstraction for in-memory cluster computing. In Proceedings of the USENIX Conference on Networked Systems Design and Implementation (NSDI '12), pages 2-2. USENIX Association, 2012.

[45] M. Zhang, X. Zhang, X. Zhang, and S. Prabhakar. Tracing lineage beyond relational operators. In Proceedings of the 33rd international conference on Very large data bases, pages 1116-1127. VLDB Endowment, 2007. 